\title{
Tratamiento de los aneurismas del seno de valsalva. A propósito de 2 casos.
}

Treatment of sinus of Valsalva aneurysms. Report of two cases.

\author{
ASTE Hernán, BRACAMONTE Luis, AlCANTARA Carlos, TALLEDO Oscar, \\ MALPARTIDA Bertilo, BASSINO Mauricio, RUIZ Fernando
}

\section{INTRODUCCIÓN}

Los aneurismas de los senos de Valsalva (ASV) obedecen a la definición general de "Aneurismas".

En este artículo presentamos los dos últimos casos que nuestro servicio, Cirujanos Torácicos y Cardiovasculares Asociados ha estudiado y tratado quirúrgicamente.

La finalidad de este trabajo, es mostrar las diferentes presentaciones de esta patología, la variabilidad de técnicas quirúrgicas, el enfoque y manejo del cirujano, y la factibilidad en nuestro medio de corregirlo exitosamente.

\section{Casos clínicos}

Caso $N^{\circ}$ 1: Mujer de 43 años de edad, procedente de Cuajote (Sur del Perú), con antecedente de un "problema cardíaco" 24 años antes, no bien referido. Cinco meses antes de su internamiento refiere cuadro de infarto miocárdico no demostrado. Llega con cuadro de angina pectoris, palpitaciones, disnea a los medianos esfuerzos, y edema vespertino de miembros inferiores. Recibía tratamiento con digoxina $0.25 \mathrm{mg} /$ día y ácido acetilsalicílico $325 \mathrm{mg} /$ día. Su prueba de esfuerzo fue negativa para esquemia miocárdica. Al examen clínico el hallazgo más importante fue un soplo sistólico de grado IV/VI en área aórtica con irradiación supraesternal. El electrocardiograma mostró boqueo incompleto de la rama izquierda que se hacía completo en la prueba de esfuerzo. En la radiografía de tórax se observó aumento de área cardiaca a expensas de AI y VI. El estudio ecocardiográfico doppler evidenció estenosis aórtica calcificada con gradiente máximo de $60 \mathrm{~mm} \mathrm{de} \mathrm{Hg}$ y regurgitación aórtica leve con pequeña regurgitación mitral y tricuspídea, hipertensión pulmonar discreta, y arco aórtico tortuoso. En el cateterismo cardiaco se evidenció, anillo valvular aórtico pequeño que impidió el ingreso a VI para medir gradiente ventrículo/arterial y no se observaron lesiones coronarias; la presión en aorta fue de 140/80 mm Hg, la presión en cuña pulmonar de $20 \mathrm{~mm} \mathrm{Hg}$, e a.pulmonar de 35/15 (27) y dilatación del seno de Valsalva izquierdo de características aneurismáticas de 
aproximadamente de 3 x $4 \mathrm{~cm}$ y con depósitos de calcio en el anillo y cuello. Con estos hallazgos se le programó para cirugía correctiva.

Caso $\mathbf{N}^{\circ}$ 2: Niño varón, de 11 años de edad con $26.2 \mathrm{Kg}$ de peso, y $1.42 \mathrm{~m}$ de estatura, con antecedente de cirugía de triple ligadura de ductus arterioso a la edad de 3 años y soplo cardiaco post operatorio por problema valvular a ser posteriormente evaluado. Tenía historia de disnea a medianos esfuerzos y palpitaciones. Al examen encontramos un paciente con tórax deformado por el impulso cardiaco (tórax en "quilla"), adelgazado y gran frémito precordial. Se le indica estudio ecocardiográfico doppler que mostró hipertrofia biventricular, CIV subaórtico infundibular, insuficiencia aórtica severa y ASV derecho roto a VD. El cateterismo cardiaco mostró estenosis pulmonar infundibular leve, insuficiencia aórtica moderada y CIV subvaluar amplia con vaciamiento rápido a ambos cámaras ventriculares muy similar a cateterismo realizado un año antes. Los valores de presión fueron: $\mathrm{Ao}=105 / 50 ; \mathrm{VI}=105 / 0-10$; A. Pulmonar=52/20 (30); $\mathrm{PCP}=12 \mathrm{~mm} \mathrm{de} \mathrm{Hg}$. No se observaron malformaciones coronarias. En estas circunstancias se programa para cirugía de ventriculoseptoplastía y reparación del ASV roto a VD.

\section{Tratamiento quirúrgico:}

Caso 1: La técnica quirúrgica se inició en la forma convencional para los cambios valvulares aórticos: Heparinización sistémica, canulación de aorta, y de cava única, entrada en circulación extracorpórea, clampaje aórtico y paro cardioplégico cristaloide fría. Luego, aspiración de cavidades izquierdas con cánula a través de vena pulmonar superior derecha, colocación de suero frío en cavidad pericárdica, aortotomía transversa y evaluación in situ.

Al tratarse de una válvula aórtica estenosada, calcificada y bicúspide, se hizo el cambio valvular convencional, colocándose una prótesis de Starr-Edwards (de bola y canastilla) $\mathrm{N}^{\circ}$ 9; pero cuyos puntos de sujeción en "U" y separados, entraban por el anillo aórtico de abajo para arriba en la forma que suele hacerse a nivel del seno de Valsalva derecho y no coronario; pero a nivel del seno izquierdo. Estos puntos se pasaban por el anillo y luego entraban nuevamente para coger el borde libre de la dilatación aneurismática plicando de esta manera dicha cavidad (Figura $\mathrm{N}^{\circ} 1$ ) (1). Los puntos fueron dados con hilo trenzado tipo Mersilene 2/0 reforzados con parches de teflón. La bolsa aneurismática quedaba entonces anulada y con los mismos puntos se anclaba la prótesis a este nivel. Se bajó la prótesis asegurando que los ostiums coronarios queden libres del anillo de sutura valvular. Finalmente, aortorrafía con prolene 4/0 en surget contínuo doble plano; se retira el aire de cavidades para evitar la embolia aérea, recalentamiento y desclampaje aórtico con recuperación de latidos normales. 


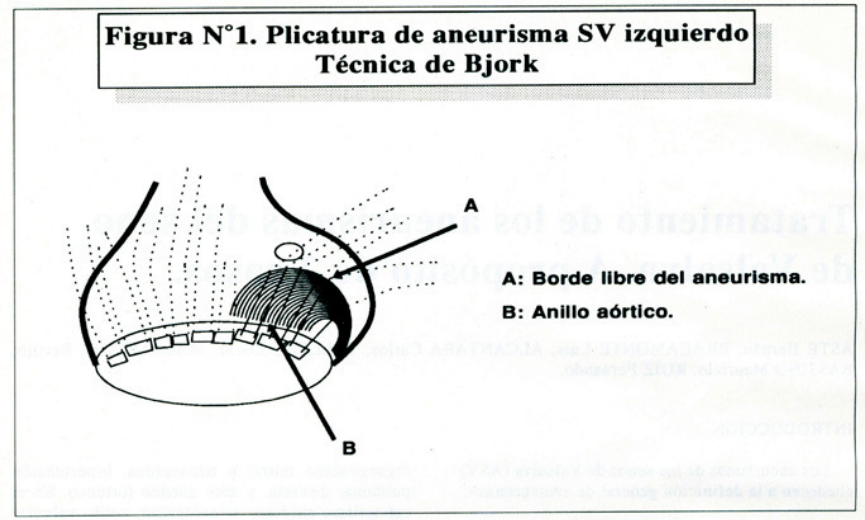

Caso 2: El abordaje de este paciente fue por toracotomía media transesternal y pericardiotomía longitudinal. Se identifican las estructuras, encontrando una gran cardiomegalia a expensas de ambos ventrículos. La raíz de la arteria pulmonar era de mayor diámetro que la raíz aórtica. Se realizó heparinización sistémica, canulación de aorta, canulación de cavas superior e inferior por separado, y se colocaron pasadores para lazar ambas venas. Seguidamente, ingreso en circulación extracorpórea, pinzamiento de aorta, enfriamiento del paciente a $28^{\circ} \mathrm{C}$, para cardioplégico cristaloide frío a través de raíz de aorta y descompresión de cavidades izquierdas con aspirador a través de aurícula izquierda. Se aspira cardioplegia a través de seno coronario en aurícula derecha abriendola parcialmente. Solución fría tópica en pericardio. Aortotomía transversa. Se observó anillo aórtico pequeño y seno de Valsalva derecho prolapsado hacia ventrículos.

Para la corrección de usó la técnica de Trusler (2), (Figura $\mathrm{N}^{\circ} 2$ ), que consiste en realinear con un punto las 3 cúspides valvares y se identificó la zona redundante del saco aneurismático plicando el exceso a nivel de las comisuras con la pared aórtica anclándolo con un punto de monofilamento y teflón, para luego reforzar con otro parche de teflón uniendo los bordes redundantes comisurales del seno dilatado con el seno adyacente. En el intraoperatorio no se confirmó ruptura del seno a VD, pero sí prolapso a esa cavidad produciendo gran insuficiencia. Luego de comprobar su competencia mediante la aortorrafía parcial y la apertura del clamp aórtica, manteniendo la tensión de las valvas con el primer punto de alineamiento, se procedió a realizar una ventriculotomía derecha transversal para el cierre del CIV, el cual estaba debajo del seno coronario derecho, con un parche de dacrón de la forma convencional, retirando el punto antes descrito. Se tuvo la precaución de no lesionar ramos coronarios durante la ventriculotomía. Se procedió a completar el cierre de aorta y ventrículo derecho, recalentamiento del paciente, retiro del aire y desclampaje aórtico para la recuperación de los latidos normales.

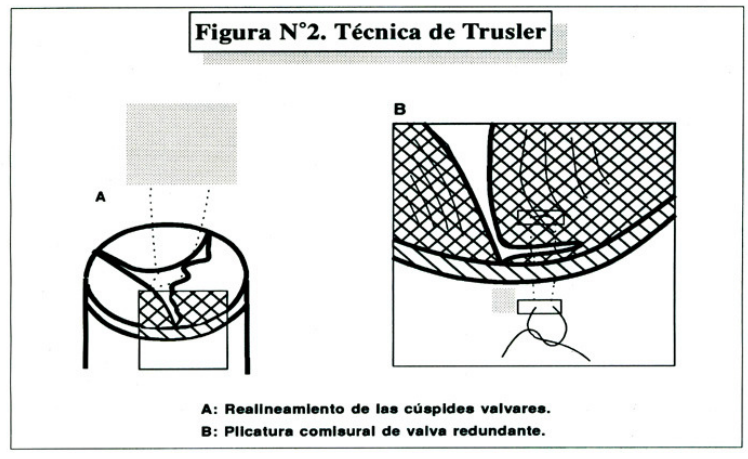




\section{DISCUSIÓN}

La evolución de los dos casos aquí presentados ha sido favorable, a excepción de una taquicardia supraventricular pasajera en el primer caso, que cedió con la reposición de líquidos y digital; y en el segundo, episodios de taquirritmias y extrasístoles supraventriculares con aberrancia tratados con diversos antiarrítmicos y reposición de potasio; se inició también, el uso de inhibidores de la enzima convertidota saliendo de alta en buenas condiciones. Ambos pacientes han sido seguidos para ver su evolución ( 20 y 8 meses respectivamente). Hasta la fecha de esta impresión, enero de 1993, ambos pacientes gozan de una vida activa. La primera paciente, se mantiene con anticoagulación oral. La prótesis valvular evaluada por ecocardiografía se mostró normal; clase funcional I de la NYHA. El segundo paciente, ha ganado peso y estatura, hay disminución del área cardiaca y desaparición del frémito precordial a pesar de mantener un leve soplo diastólico de insuficiencia aórtica que está siendo controlado por ecocardigrafías repetidas.

El éxito depende de la etiología asociaciada a otras patologías; depende de uno, otros o varios senos comprometidos; de la urgencia de la cirugía; del estado previo del paciente; de su situación socio-económica; y por último, de la experiencia del equipo quirúrgico. La frecuencia en nuestro grupo es de 5 pacientes en aproximadamente 20 años, lo que hace el $0.23 \%$ de todas nuestras cirugías, semejantes a las estadísticas de la literatura.

En los 2 casos que presentamos se utilizaron 2 técnicas diferentes ya que eran alteraciones también diferentes: Plicatura para el caso de estenosis aórtica con ASV izquierdo; y la técnica de Trusler para el caso del prolapso aneurismático y CIV. Localizaciones diferentes, etiologías diferentes, diferentes técnicas de reparo, otros abordajes, otras metas. En el primer caso la corrección de la estenosis aórtica que sobrecarga el ventrículo izquierdo era la principal meta, y la plicatura del ASV, probablemente consecuencia de la patología anterior era un punto importante, pero no crítico en el momento de la cirugía. La corrección fue un cambio valvular por una prótesis mecánica aprovechando el gesto quirúrgico para corregirle el aneurisma, no siendo posible una cirugía conservadora.

El segundo caso difiere por tratarse de un niño, con patología congénita asociada y con insuficiencia aórtica, y en el cual se iba directamente a corregirle el propio aneurisma, que se suponía roto por la ecocardiografía, y cerrar la comunicación interventricular. La técnica de Trusler permite mantener la válvula nativa del paciente; evita de esta forma que el paciente tome anticoagulantes, y también una reoperación casi segura si se utilizan prótesis mecánicas. Algunos de estos pacientes quedan con una pequeña insuficiencia que no es hemodinámicamente significativa, pero que requieren controles frecuentes tanto clínicos como ecocardiográficos (3).

Algunos autores prefieren limitarse a cerrar la comunicación interventricular en los casos en que el aneurisma no está roto; o hay quienes cierran la CIV y plican el aneurisma con otro parche de dacrón (4). Otra técnica muestra la plastía del velo aórtico previa resección de la porción dilatada incorporando el borde de sección a la sutura del defecto ventricular. Y así existen otras técnicas muy útiles. 
Agradecimientos:

Por su gentil e invalorable colaboración a los Dres. Tapia Fernando, Vargas Mario, Bustamante Guillermo, Castañeda Aldo, Zamalloa Oscar, Vargas Willy y Laura Manuel.

\section{Correspondencia:}

Dr. Hernán Aste

Av. Guardia Civil \# 333

Lima 41 Lima, Perú

Teléfono y Fax (5114) 752988

\section{BIBLIOGRAFÍA}

1.Bjork VO, Bjork L. Aneurysm of sinus of Valsalva. J Thorac Cardiovasc Surg 1965; 50:16-21

2.Kirklin JW, Barratt-Boyes BG. Cardiac Surgery, 1986. Nueva York, U.SA.

3.Trusler GA, Williams WG, Smallhorn JF, et al. Late Results after repair of Aortic Insufficiency associated with ventricular septal defect. J Thorac Cardiovasc Surg 1992; 103:276-81

4.Bonhoeffer P, Fabbrocini M, Lecompte Y, et al. Infundibular Septal Defect with severe aortic Regurgitation: A New Surgical Approach. Ann Thorac Surg 1992; 53: 857-3. 